\title{
Numerical Analysis on Crack Tip of Fracture Splitting Groove Stress-strain Field of Main Bearing Block of Engine
}

\author{
Dan Yang ${ }^{1, a}$, Yuheng Ning ${ }^{2, b}$ \\ ${ }^{1}$ Jilin Agricultural University, Changchun, 130118, China \\ ${ }^{2}$ Datang Northeast Electric Power Test\& Research Institute Co.,Ltd, Changchun, 130118, China \\ a,bemail: 1228256215@qq.com
}

Keywords: Engine Crankcase; Fracture Splitting; Fracture Splitting Groove; Stress-strain Field

\begin{abstract}
The parameters of fracture splitting groove have direct influence on the stress-strain field of crack tip. This paper modeled on some cylinder engine crankshaft bearing bracket. Based upon normal stress criterion of cleavage fracture and the mechanics of fracture software ABAQUS, the stress-strain field of different parameters were simulated and analysed, and the effect of parameters on cracking process were investigated using cleavage fracture criterion. The results indicated that the material of crankcase is fragile material. Although the material is not sensitive to gap, but on account of crack initiation, crack growth, machining of fracture splitting groove and fracture splitting load, optimum parameters for fracture splitting groove were selected. The experiment with optimized parameters made excellent results.
\end{abstract}

\section{Introduction}

The car engine is a key part of vehicles provided driving force, and it directly affect acceleration performance, economy, and environmental protection. With the development of car industry, the manufacturing processes of car engine has great progress e.g. the emergence of fracture splitting. By nature, due to brittleness, the initial crack source serves as microcracking induced by perpendicular normal stress on it. It is developed that the process is characterized by short processing chain, high accuracy, low equipment investment, and low production cost. The fracture splitting technology is well developed. As a typical split part, the engine crankshaft bearing bracket has a similar structure to connecting rod: the main structure and bearing cover are assembled by screws. However, the structure materials are different in the two cases.

The initial cracking source of fracture splitting is the fracture splitting groove. As inferred from the theoretical analysis and studies on connecting rod, the strain-stress field of crack tip is significantly affected by parameters of the fracture splitting groove. Therefore, in this work, ABAQUS software is employed to simulate the cracking process of some cylinder engine crankshaft bearing bracket. To obtain the corresponding optimized parameters with splitting experiments, the influence of parameters of fracture splitting groove on the strain-stress field of crack tip is analyzed.

\section{Mechanism and Criterion of Brittle Fracture}

In order to keep the conformity of the fracture as well as the small deformation of the arc surface during fracture splitting, cleavage fracture should be conducted. As a brittle fracture, rapid separation along cleavage boundaries is led by failure of atomic bond due to the normal stress on metal. In the case of cleavage fracture for notch, two conditions needs to be met, as shown in Fig. 1. a critical status of plastic strain, $\varepsilon p \geqslant \varepsilon$ pc, where $\varepsilon p, \quad \varepsilon$ pc stand for the strain of notch and limit strain of the material, which leads to the formation of the splitting nuclei. On the other hand, to ensure cracking after nuclei form, $\sigma$ yy $\geqslant \sigma \mathrm{f}$, namely the main stress outreaches the cleavage fracture stress. In addition, an extra condition is shown: $\sigma_{\max } / \bar{\sigma} \geq T_{C}$, namely the ratio of the maximum stress at the notch to the equivalent stress to ensure that cracking cannot be deactivated. 
For the same material, $\varepsilon$ pc and $\sigma \mathrm{f}$ are stable, and they are insensitive to the loading rate, the shape of the notch and temperature. So the nucleation and propagation of cracks are mainly affected by $\varepsilon \mathrm{p}$ and $\sigma \mathrm{yy}$. As the sheet thickness is given constant, the notch is determined by $\mathrm{a}, \mathrm{h}$ and $\mathrm{r}$, namely flare angle, groove depth and the radius of curvature, respectively. As shown in previous studies, with the same load, different parameters of the notch, such as the normal stress $\sigma$ yy, the ratio of three dimensional stress $\mathrm{T}\left({ }^{\sigma_{\max } / \bar{\sigma}}\right)$ and plastic strain $\varepsilon \mathrm{p}$, have different values. Therefore, it is important to design and optimize the parameters of notch of the crankshaft bearing bracket, which weighs a lot for fracture splitting on cylinder engine.

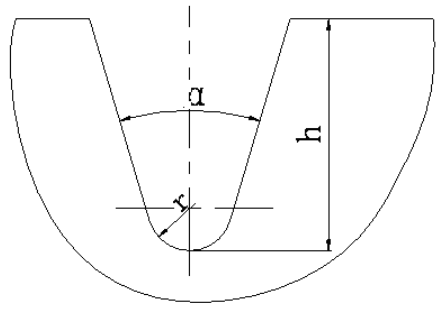

Fig.1 Notch parameter

\section{Modeling and Material Characterization}

\section{Three-dimensional Modelling.}

In this work, during fracture splitting, the same load was added to each bearing block of cylinder engine crankshaft bearing bracket and thus all the bearing bracket fractured simultaneously, so only one bearing block was studied. Fig. 2a gives the three dimensional model of the bearing block. It can be noted here, the crack propagation is not a topic, hence the effect of the screw hole on fracture splitting is not considered. At first the bearing block in Fig. 2a is divided by the longitudinal symmetrical axis. Then half of it is divided again by the symmetrical axis of thickness, which is acquired in Fig. 2b. The diameter of the hole is $59 \mathrm{~mm}$, the diameter of the external arc is $100 \mathrm{~mm}$. So the maximum width between the two sides is $100 \mathrm{~mm}$. The distance between the two longitudinal screw holes is $76 \mathrm{~mm}$, the diameter of which is $10 \mathrm{~mm}$. The thickness of the bearing block is $21 \mathrm{~mm}$.
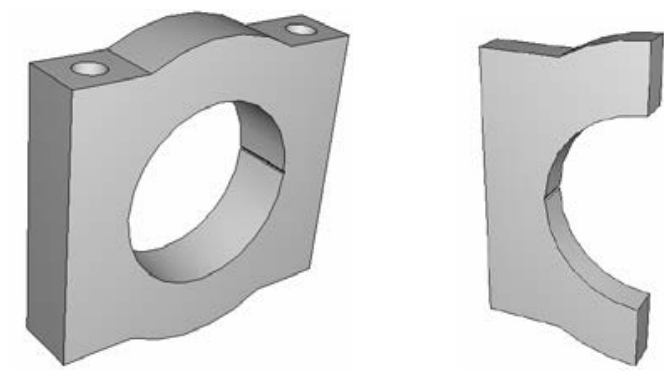

Material Characteristics.

a A single bearing

b $1 / 4$ model

Fig. 2 Three-dimensional model of engine crankcase

In this work, the structural material of the cylinder engine crankshaft bearing bracket is gray cast iron. The elastic modulus is $1.018 \times 10^{5} \mathrm{Mpa}$; Poisson ratio $(\gamma)$ is 0.227 ; normal yield stress $\left(\delta_{0.2}\right)$ is 113.4 MPa; fracture stress $(\delta)$ is $154.12 \mathrm{MPa}$.

\section{Effect of Fracture Splitting Groove Parameters on the Stress-strain Field of the Crack Tip}

During fracture splitting, it is important to create a gap, thus leading to stress concentration and then crack propagates from the gap. The structural material of cylinder engine crankshaft bearing bracket is brittle. So during fracture splitting, tensile failure is the final result of tensile stress at the gap. Thereby crack initiation can be determined by the maximum normal stress criterion. The 
forefront of the gap with the maximum normal stress is easier to crack.

\section{Effect of the Splitting Groove Flare Angle on the Stress-strain Field of the Crack Tip.}

In this work, two fracture splitting parameters, the radius of curvature (r) and the depth (h), are set consistent as $0.2 \mathrm{~mm}$ and $0.6 \mathrm{~mm}$, respectively. Normal load was given against the splitting arc. As the normal load was increased up to $30 \mathrm{MPa}$, the principal stress at the crack tip $\sigma_{\text {yy }}$ reached the value of fracture. So the relation of the above mentioned parameters with the normal stess ranging from 10-30 MPa is studied.

According to the fracture equation of the bearing block and the three criterion of cleavage fracture, the maximum normal stress $\sigma_{y y}$ plays a key role in the parameters of crack propagation after crack initiation. Fig. 3 shows that when the flare angle ranges from 45-90 degrees, only mild change of the maximum normal stress can be acquired, and the peak value at the flare angle is $60^{\circ}$. However, the change is mild in general. The equivalent plastic strain for crack initiation reached similar high values at the flare angles of $45^{\circ}$ and $60^{\circ}$, respectively. The three-dimensional stress for crack nuclei and growth is stable under various loads. Unlike C70S6, the structural material of contacting rod, HT150, the structural material of cylinder engine has poor gap sensitivity. So crack nucleation results in the following processes. On the other hand, with regard to relatively small flare angle and inconvenience for the tool change, the optimal value for the splitting groove flare angle is set as $60^{\circ}$. This condition is not very strict: $60^{\circ}$ would be favorable yet values nearby it are also acceptable.

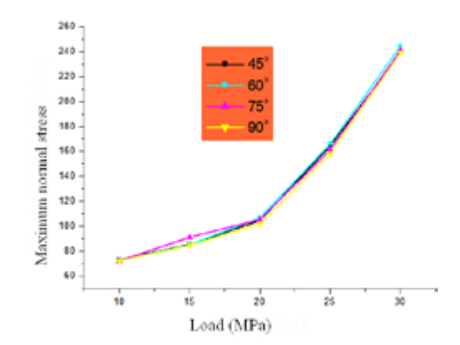

a Maximum normal stress (MPa)

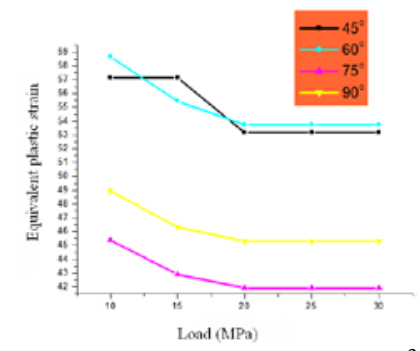

b Equivalent plastic strain $\left(10^{-3}\right)$

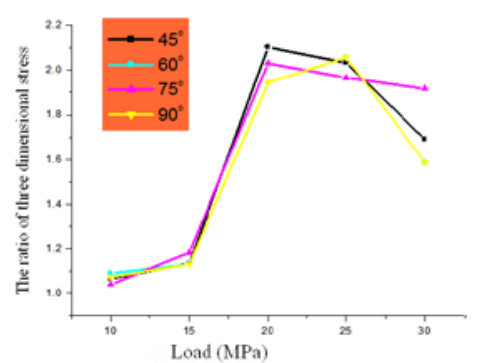

c The ratio of three dimensional stress

Fig.3 The changes of the parameters with notch field angle under different loads

\section{Effect of the Gap Depth on the Stress-strain Field of the Crack Tip.}

With regard to fracture splitting of connecting rod, parameters in this work were set as: gap depth $0.4-0.7 \mathrm{~mm}$, flair angle of the splitting groove $\alpha 60^{\circ}$; radius of curvature of the splitting groove r $0.2 \mathrm{~mm}$, respectively. Curves of variables with various loads were illustrated in Fig. 4.

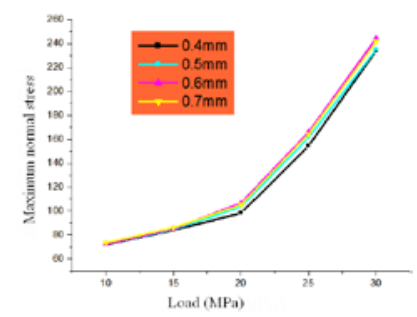

a Maximum normal stress (MPa)

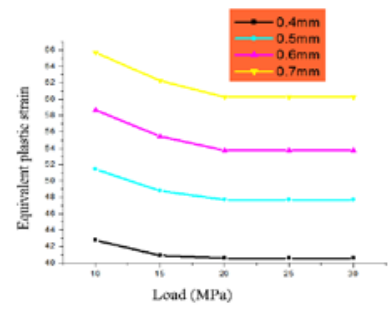

b Equivalent plastic strain $\left(10^{-3}\right)$

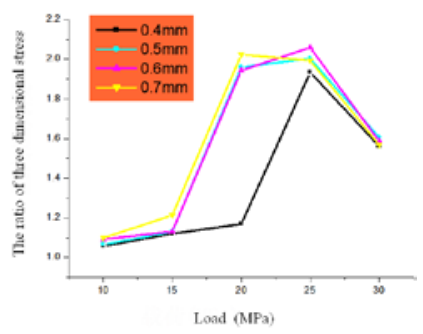

c The ratio of three dimensional stress

Fig. 4 The changes of the parameters with notch depth under different loads

Furthermore, the effect of the gap depth on the stress-strain field of the crack tip was analyzed. The difference in the maximum normal stress at the crack tip with the depth range of $0.4-0.7 \mathrm{~mm}$ is mild. The difference in three-direction force exhibits similar results. The difference in the equivalent plastic strain is relatively large, affecting crack nucleation. Due to high content of graphite and poor gap sensitivity of gray cast iron, the deeper it reaches, the easier the nucleation is. With process conditions and tool costs taken into account, the splitting groove would be bored off at the end of this process. However, the bore depth should be given properly. Some previous studies 
show that the increasing depth of the splitting groove can effectively lower the working load. So a rational value of the depth is usually set as $0.6 \mathrm{~mm}$.

\section{Effect of the Radius of Curvature on the Stress-strain Field.}

The radius of curvature was studied with splitting flair angle $\alpha o f 60^{\circ}$ and depth h of $0.6 \mathrm{~mm}$. The study range of radius curvature is $0.1-0.3 \mathrm{~mm}$ with various loads. Fig. 5 presents the test results with different radii of curvature.

In Fig. 5a, the maximum normal stress increases with the improving load after crack nucleation. The increase trend of the maximum normal stress, at the radius of curvature of 0.1 , is more obvious than the cases with the radius of curvature of the other values, indicating that the stress concentration at the radius of curvature of 0.1 is more obvious than the cases with the radius of curvature of the other values indicating that crack initiation can take place easier. In Fig. 5b, higher value is acquired for the equivalent plastic strain $\varepsilon_{\mathrm{PEEQ}}$ at the radius of curvature of 0.1 than the cases with the radius of curvature of the other values, which means that crack nucleation can take place easier at the gap. In Fig. 5c, when the load reached up to $22 \mathrm{MPa}$, the three direction force at the radius of curvature of 0.1 is higher than the cases with the radius of curvature of the other values, indicating that crack propagation can progress easier. Depending on the results above, it can be concluded that the structural material of bearing block is more sensitive to the radius of the curvature than to the other two variables. So smaller radius of curvature is preferred for fracture splitting. In general, two methods are used to process the splitting groove: laser treatment and pulling. In the case of pulling, the smaller radius of curvature leads to worse tool consumption. So the preferable value for the radius of curvature is set as $0.1 \mathrm{~mm}$. In the case of laser treatment, it can be set as $0.05 \mathrm{~mm}$.

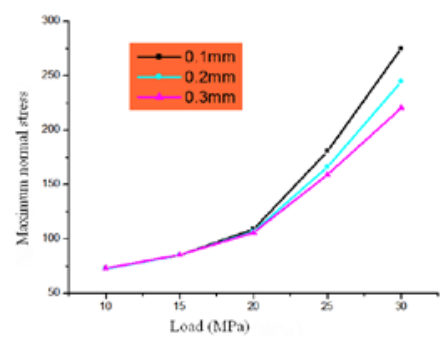

a Maximum normal stress ( $\mathrm{MPa}$ )

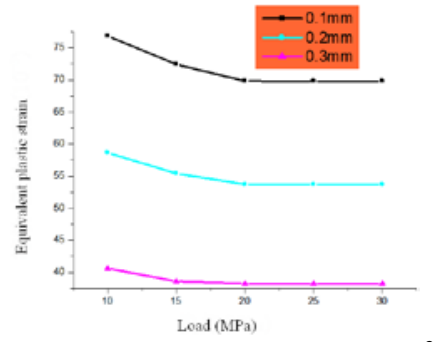

b Equivalent plastic strain $\left(10^{-3}\right)$

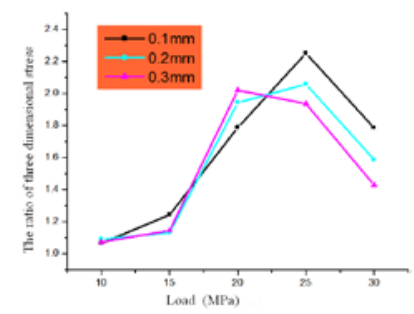

c The ratio of three dimensional stress

Fig 5. The changes of the parameters with notch curvature radius under different loads

\section{Results and Discussion}

In order to verify the above simulation, a mechanical tester CSS-88300 was employed to conduct fracture splitting. The maximum nominal tension is $300 \mathrm{KN}$. The frequency of data collection is 200 $\mathrm{Hz}$. As aforementioned, the radius of curvature is the dominant variable. Consequently the following parameters were designed:

The maximum loads for fracture splitting are $97 \mathrm{KN}, 118 \mathrm{KN}$ and $126 \mathrm{KN}$, respectively. The breaking effects with the former two values are better than the latter, with mild plastic strain acquired. However, when the radius of curvature reached up to $0.1 \mathrm{~mm}$, the lowest value of splitting load was obtained, with smallest plastic strain of all the all samples. In addition, the splitting result in this case appeared to be the best amongst the all samples. So based on the test results, the following parameters are preferable: $\alpha=60^{\circ}, h=0.6 \mathrm{~mm}$, and $r=0.1 \mathrm{~mm}$, respectively.

Tab. 1 The notch geometry parameters of test pieces

\begin{tabular}{c|c|c|c}
\hline & $\begin{array}{c}\text { first } \\
\text { set }\end{array}$ & $\begin{array}{c}\text { Second } \\
\text { set }\end{array}$ & $\begin{array}{c}\text { Third } \\
\text { set }\end{array}$ \\
\hline groove depth $(\mathrm{mm})$ & 0.6 & 0.6 & 0.6 \\
\hline flare angle $\left(^{\circ}\right)$ & 60 & 60 & 60 \\
\hline radius of curvature(mm) & 0.1 & 0.2 & 0.3 \\
\hline
\end{tabular}




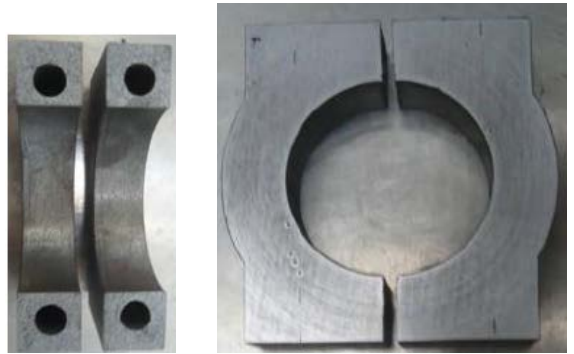

Fig 6. Splitting testing piece

\section{Conclusions}

In this work, fracture splitting of the cylinder engine crankshaft bearing bracket made of gray cast iron HT150 was simulated and then carried out using a mechanical tester. The following conclusions are obtained:

1. It is shown that little effect of flair angle of the splitting groove on the stress-train field of the crack tip is shown. With respect to working conditions, the value of $60^{\circ}$ is preferable.

2. Mild effect of the splitting groove depth on normal stress is acquired. However, the deeper it reaches, the easier the crack nucleation turns. In the case that the splitting groove would be bored off later during splitting, $0.6 \mathrm{~mm}$ is the preferable value for this variable.

3. Amongst all three variables, the radius of curvature presents the maximum influence on the stress-strain field of the crack tip. Smaller radius of curvature makes crack nucleation and crack propagation easier. With respect to the tool consumption, $0.1 \mathrm{~mm}$ is the preferable value for this variable.

The test results are congruent with the simulation, verifying the effectiveness of the simulation and thus shedding light on parameter design of fracture splitting of cylinder engine crankshaft bearing bracket.

\section{References}

[1] http://muzhi.baidu.com/question/477155699.html

[2] Kou Shuqing, Jin Wenming, etc. The New Technology and Developing Trend of IC Engine Connecting Rod Manufacturing [J]. Chinese Internal Combustion Engine Engineering.2001.

[3] Yang Shenhua, Kou Shuqing, ZhaoYong, etc. Processing method and assembly for fracture splitting of main bearing cap of engine crankcase bearing bracket. china. 200410010822.4[P]. 2005-05-04.

[4] Wang GZ,Chen J H. Cleavage fracture criterion of low alloy steel and weld metal in notched specimens [J ] . International Journal of Fracture,1998 (89).

[4] Fetouh, Mohamed A. Method for making a split bearing assembly[P]. United States Patent:4569109.

[5] Huang Mingzhi. Mechanical properties of metals[M]. Xi'an: Xi`an Jiaotong University Press,1986 